\title{
Éditorial
}

\section{LA CORROSION HIER ET AUJOURD'HUI...}

\section{Un siècle de corrosion}

C'est à partir du $19^{\mathrm{e}}$ siècle que l'on se préoccupe de distribuer, par des conduites, de l'eau en grande quantité et sur des sites de plus en plus nombreux. On utilise alors des matériaux bon marché, simples à fabriquer, censés résister à la corrosion et faciles à mettre en œuvre. Ainsi trouve-t-onau départ de l'ère industrielle deux matériaux de prédilection: la fonte grise à graphite lamellaire et le plomb. Plus tard, le développement des techniques permettra l'émergence de l'acier sous forme de tubes en acier noir ou en acier galvanisé tandis qu'on verra, pour les petits diamètre, l'essor du cuivre. Les aciers inoxydables et les alliages d'aluminium apparaîtront au $20^{\circ}$ siècle mais leurs applications resteront très limitées dans le domaine de la distribution de l'eau.

A cette époque, le problème de la corrosion ne se pose que dans la mesure où les installations doivent assurer leur fonction, c'est à dire ne pas percer et ne pas laisser apparaître trop de fuites. L'action du matériau sur l'eau est un paramètre secondaire. Bien sûr on connaît des cas où le plomb peut conduire au saturnisme, mais on se rassure en pensant que cela ne concerne que les eaux douces peu «calcaires» et l'on pense que dans les eaux plus dures le plomb est recouvert de calcaire et qu'ainsi le consommateur est protégé. 
Ce n'est que plus tardivement que l'on va se poser le problème de la compatibilité des matériaux avec l'eau distribuée. Au cours du siècle passé les problèmes de corrosion des métaux ont bien entraîné quelques dégâts, mais l'apparition des matériaux organiques a un temps laissé penser aux exploitants que ces nouveaux matériaux pouvaient prendre la relève, soit comme constituants exclusifs des canalisations ou appareillages, soit comme revêtements interne des structures métalliques. Ces produits étant nouveaux, et d'origine organique, on s'est tout de même posé la question de la toxicité éventuelle des matières relarguées par ces matériaux. Les travaux les plus récents tendent à démontrer qu'il ne s'agissait pas d'un faux problème. Et, parallèlement, on en est venu progressivement à se poser les mêmes questions en ce qui concerne les matériaux métalliques.

Aujourd'hui, sur les réseaux publics récents, l'eau n'est plus guère en contact avec des matériaux métalliques : la fonte comme l'acier sont revêtus de ciments ou de polymères. Les autres conduites sont à base de ciments ou de matières plastiques. Cependant il n'en est pas de même dans les réseaux privés où subsistent du plomb mais surtout de l'acier galvanisé et du cuivre. Aussi le problème de la corrosion se trouve posé de manière tout à fait nouvelle par rapport avec ce que l'on avait l'habitude de faire depuis environ un siècle.

Même si les chimistes et les applicateurs mettent à la disposition des réseaux et des usagers des matériaux sans cesse améliorés, il n'empêche que les métaux ont encore un bel avenir. Pour des raisons de sécurité certaines installations se doivent d'être métalliques. Les caractéristiques mécaniques des métaux sont tout de même très supérieures à celles de la plupart des polymères ou composites usuels. Cependant, si on doit continuer à utiliser les métaux, on sera de plus en plus exigeant en ce qui concerne le relargage d'ions métalliques et de produits de corrosion. Tout cela invite à porter un nouveau regard sur la manière d'étudier les diverses formes de la corrosion.

\section{Pourquoi l'eau corrode-t-elle?}

Dans la pratique, il n'y a pas d'eaux types, mais des eaux particulières. Du point de vue de la corrosion, on pourrait croire que l'on se place alors dans le cas de fluides corrosifs «simples», compte tenu du caractère banal de ce type de milieu. En fait, il n'en est rien, car ces électrolytes aqueux présentent des particularités qu'il est nécessaire de bien comprendre pour éviter tout incident de fonctionnement. 
Une eau ordinaire est en général peu minéralisée, la quantité de sels dissous est souvent inférieure à $1 \mathrm{~g} / \mathrm{l}$, et plus rarement comprise entre 1 et $2 \mathrm{~g} / \mathrm{l}$. Cela veut dire que la corrosivité propre d'un tel milieu est assez faible. Mais la plupart des eaux contiennent de l'oxygène dissous et c'est cet élément qui est alors l'agent corrosif principal. En outre, comme la minéralisation d'une eau est relativement faible, les réactions de corrosion peuvent entraîner facilement des variations de composition importantes dans des zones où le fluide est peu renouvelé. Ainsi, l'eau initiale qui a pénétré dans une anfractuosité se transforme plus ou moins rapidement, sous l'effet de la corrosion, en acide chlorhydrique. On comprend aisément que la corrosivité se trouve alors fortement accrue. Elle va bien sûr dépendre du milieu initial, mais surtout de la nature du métal et des conditions d'exploitation du système. Avec du fer ou de l'acier noir on pourra atteindre des $\mathrm{pH}$ de l'ordre de 2 tandis qu'avec du cuivre on peut obtenir un pH de 3,5 en fond de piqûre. Les aciers inoxydables, quant à eux, donnant des $\mathrm{pH}$ encore plus bas. C'est pourquoi, on ne pourra parler de corrosivité d'une eau que vis-à-vis d'un métal donné, tout en sachant que cette corrosivité n'expliquera pas à elle seule toutes les corrosion rencontrées.

\section{Comment aujourd'hui appréhender correctement les phénomènes de corrosion.}

A l'origine la corrosion est comprise comme la dissolution d'un métal dans un électrolyte. Tout l'art des corrosionistes va donc être de caractériser la vitesse de disparition du métal au cours du temps, vitesse que, la plupart du temps, il va exprimer en perte d'épaisseur annuelle du matériau. Bien sûr on s'apercevra, très tôt, que l'essence de la corrosion en milieu aqueux est d'être un phénomène de type électrochimique qui associe une oxydation (celle du métal) à des réductions (celles d'espèces de l'électrolyte). C'est pourquoi en matière de corrosion, les électrochimistes ont progressivement pris la relève des métallurgistes. L'apparition, dans les années 50 des matériels électroniques destinés aux études électrochimiques a ensuite entraîné une quasi hégémonie de l'électrochimie en corrosion. Or, les chercheurs soucieux d'utiliser au mieux leurs techniques ont toujours cherché à éviter les systèmes qui ne "marchaient pas bien» comme par exemple le cas du fer ou du cuivre dans l'eau. Pour avoir des résultats exploitables (« de belles courbes») la pente naturelle de l'expérimentateur l'entraînait à modifier le métal et surtout l'électrolyte pour arriver à avoir un système simple et reproductible. Insensiblement on glissait du couple fer/eau naturelle vers le couple fer/solution de chlorure de sodium à $\mathrm{pH}=1$, et désaérée. Ainsi on arrivait à un système où la réaction anodique était bien celle de 
l'oxydation du fer mais où la réaction cathodique était la réduction des protons solvatés $\mathrm{H}+$ en hydrogène gazeux. L'ajout de $\mathrm{NaCl}$, lui, augmentait la conductivité ce qui entraînait, en conséquence, des densités de courant aisément mesurables. Mais la réalité est tout autre. Aujourd'hui il faut être conscient que le couple métal/électrolyte n'existe pas. En fait la corrosion met en jeu des interactions complexes sur plusieurs niveaux:

- un niveau qui est celui de l'interface métal/produits de corrosion

- un niveau qui correspond à l'interface produit de corrosion/biofilm

- un niveau qui se situe à l'interface biofilm/couche limite de l'écoulement

- un niveau qui est celui que l'on trouve entre la couche limite (peu renouvelée) et le liquide circulant.

Cette situation est fort peu attrayante pour l'électrochimiste «classique» car elle ne conduit pas à des choses «claires».

De plus, la complexité est encore aggravée par le fait que cette situation est évolutive dans le temps: les produits de corrosion évoluent en fonction de l'âge de l'installation et aussi en fonction des conditions initiales de leur formation. Par ailleurs la construction de l'installation, la mise en eau, ainsi que les conditions de désinfection, de rinçage ultérieur, puis les conditions d'exploitations vont jouer un rôle extrêmement important. La question qu'on peut alors se poser, c'est que faire aujourd'hui pour maîtriser au mieux les problèmes de corrosion?

\section{Comment suivre les phénomènes de corrosion dans les eaux}

La première chose qu'il faut admettre, c'est que l'on ne peut pas envisager, en matière de corrosion, d'essais de laboratoire ou sur témoins de courte durée. Le premier point à établir est de savoir si l'installation, telle qu'elle est envisagée, va subir une corrosion uniforme ou au contraire va présenter des risques de corrosion localisée. Si l'on excepte le cas des aciers inoxydables, où la corrosion par l'eau peut conduire à des percements en quelques mois, la plupart des matériaux usuels sont ruinés par la corrosion localisée à l'issue de quelques années. L'ordre de grandeur de 2 ans est relativement raisonnable. De ce fait, on est donc contraint, par la réalité même des phénomènes mis en jeu par la corrosion, de réaliser des essais sur des pilotes, ou de suivre des éléments d'une installation déjà en service, sur une période minimale de deux ans. 
A l'issue de ces deux ans d'essais, ou d'observation in situ, on pourra s'assurer que les phénomènes de corrosion conduisent bien à une corrosion uniforme. Cette corrosion n'aura pas besoin d'être quantifiée en perte d'épaisseur de matériaux car ce n'est pas cette perte d'épaisseur qui sera le paramètre contraignant. Ce qui pourra être contraignant ce sont les paramètres secondaires comme le relargage d'ions métalliques ou de produits de corrosion (cas des eaux « rouges»). Les essais et contrôles doivent donc s'attacher à mettre en œuvre les dosages nécessaires pour vérifier si les éléments relargués sont en dessous des valeurs admises par les normes en vigueur. En outre si la formation des produits de corrosion protecteurs est correcte, ces teneurs doivent baisser au cours des deux années pour ensuite se stabiliser.

\section{L’importance de la qualité des matériaux}

Les matériaux généralement employés en distribution d'eau sont ce qu'on appelle des matériaux usuels. Ceci veut dire qu'à notre époque de large ouverture des marchés, l'origine de ces produits peut être extrêmement variée. Or ces produits obéissent en général à des normes minimales surtout en ce qui concerne la composition du matériau. On devra donc être particulièrement attentif aux produits de relargage de ces matériaux, produits souvent très différents de ceux attendus. La présence de métaux lourds n'est pas à exclure, comme par exemple le plomb au niveau de raccords (où il est présent pour faciliter l'exécution des pièces élaborées par usinage) et aussi au niveau des brasures. C'est ainsi que l'on peut retrouver du plomb dans des réseaux sans plomb. Par ailleurs des pollutions, organiques, ou inorganiques, tenaces peuvent se révéler à la surface de ces matériaux.

Ainsi donc le corrosioniste d'aujourd'hui est forcément un homme très modeste qui doit rester ouvert face à l'apparition de beaucoup de problèmes imprévus. Au delà des connaissances métallurgiques et électrochimiques il doit être attentif à tous les problèmes d'analyse de pollutions superficielles. Ces pollutions peuvent en effet êtres gênantes en elles mêmes, mais aussi par le fait qu'elles peuvent modifier le processus de mise en place des produits de corrosion protecteurs et, de ce fait, conduire à des relargages métalliques plus importants que ceux que l'on pouvait observer avec des matériaux normaux. Enfin il n'oubliera pas que la corrosion d'un réseau dépendra surtout des défauts de la technologie mise en ceuvre que ce soit en matière de construction ou d'exploitation. 